\title{
Depression and sleep disturbances in patients with multiple sclerosis and correlation with associated fatigue
}

\author{
Karthik Nagaraj, Arun B. Taly ${ }^{1}$, Anupama Gupta ${ }^{1}$, Chandrajit Prasad ${ }^{2}$, Rita Christopher ${ }^{3}$ \\ Departments of Neurology, Bowring Medical College, ${ }^{1}$ Neurological Rehabilitation, ${ }^{2}$ Neuro-Imaging and Interventional Radiology, \\ ${ }^{3}$ Neuro-Chemistry, National Institute of Mental Health and Neuro Sciences, Bangalore, India
}

\begin{abstract}
Objective: To observe prevalence of depression and sleep disturbances in multiple sclerosis (MS) patients and their correlation with associated fatigue. Study Design and Setting: Prospective observation study in a university tertiary research hospital in India. Materials and Methods: Thirty-one patients ( 6 male and 25 female) with definite MS (McDonald's criteria) presented in out-patient/admitted in the department of neurology (between February 2010 and December 2011) were included in the study. Depression was assessed using Beck's Depression Inventory (BDI). Sleep quality was assessed using Pittsburg Sleep Quality Index (PSQI). Disease severity was evaluated using the Kurtzke's expanded disability status scale (EDSS). Fatigue was assessed using Krupp's fatigue severity scale (FSS). We tried to observe correlation of depression and sleep disturbance with associated fatigue in MS patients. Results: The age of patients varied between 16 and 50 years (30.1 \pm 9.1 ). The mean age at first symptom was $25.2 \pm 6.4$ years (range 14-39 years). The prevalence of sleep disturbance and depression was 51.6\% (16/31) each and fatigue 58.1\% (18/31) in the study group. The PSQI scores were significantly greater in the patients with fatigue as compared with those without fatigue indicating poorer sleep quality is associated with fatigue in MS $(P=0.005)$. The BDI scores were also significantly higher in the fatigue group showing that severity of depression also strongly correlated with fatigue $(P=0.001)$. Conclusions: Depression and sleep disturbance in patients with MS is significantly correlated with associated fatigue.
\end{abstract}

Key words: Depression, fatigue, multiple sclerosis, sleep disturbance

\section{Introduction}

Depression commonly accompanies multiple sclerosis (MS), with a prevalence of up to $50 \%{ }^{[1,2]}$ Depression itself can manifest as fatigue. Symptoms of depression are often mistaken for fatigue (loss of motivation, anhedonia), making this condition difficult to differentiate from MS-associated fatigue. Studies in the past have shown longitudinal correlation between depression and fatigue. ${ }^{[3]}$

\begin{tabular}{|l|l|}
\hline \multicolumn{2}{|c|}{ Access this article online } \\
\hline Quick Response Code: & Website: \\
\hline & www.ruralneuropractice.com \\
\cline { 2 - 3 } & \\
\hline & \\
\hline
\end{tabular}

Regardless of any contribution to fatigue, depression should be addressed and treated, as it has substantial impact on quality of life. ${ }^{[4]}$

More than $60 \%$ of patients with MS complain of chronic sleep disturbances resulting in daytime sleepiness, increasing fatigue and depression. ${ }^{[5]}$

Specific sleep disorders or problems that may affect MS patients disproportionately include restless legs syndrome, periodic limb movement disorder, chronic insomnia, and circadian rhythm disturbances. MS patients with sleep disturbance also have significantly longer sleep latency and increased use of sleep medications. Fatigue worsens in MS patients with progressive disease especially once the ambulation is affected. ${ }^{[6]}$

Risks for chronic insomnia in MS patients may be secondary to pain, spasticity, depression, anxiety,

Address for correspondence:

Dr. Anupam Gupta, Department of Neurological Rehabilitation, National Institute of Mental Health and Neuro Sciences, Bangalore - 560029 , Karnataka, India. E-mail: drgupta159@yahoo.co.in 
nocturia, medication effects, or primary sleep disorders such as restless leg syndrome or periodic limb movement disorder. Potential causes such as these must be addressed, as insomnia and poor sleep quality in MS patients correlate significantly with quality of life.

The objective of this study was to observe the prevalence of depression and sleep disturbances in MS patients and also to observe if there is a correlation between these features and associated fatigue.

\section{Materials and Methods}

This prospective study was conducted in a university tertiary research hospital in India. Thirty-one MS patients reporting to the outpatient services/admitted in the department of neurology between February 2010 and December 2011 were included in the study. Informed consent was taken from all the patients for participation. Patients with definite MS (McDonald's criteria) $)^{[7]}$ were included in the study irrespective of age and gender. Patients who had an acute relapse in the preceding 2 months, patients with systemic infections, those who had received pulse methylprednisolone during the past 4 weeks, and those who were already receiving medications for fatigue and depression were excluded from the study.

Disability in MS patients was assessed using Kurtzke's expanded disability status scale (EDSS). ${ }^{[8]}$ This scale provides a score between 0 (very little disability with a normal neurological examination) and a maximum of 10 (death due to MS). Levels 1-4.5 refer to people with a high degree of ambulatory ability and the subsequent levels 5-9.5 refer to the loss of ambulatory ability. In addition, it also provides eight subscale measurements called functional system scores. These systems include pyramidal, cerebellar, brainstem, sensory, bowel and bladder, visual, cerebral, and others (miscellaneous associated symptoms). The functional systems are scored on a scale of 0 (low level of problems) to 5 (high level of problems) to reflect the level of disability observed clinically.

Fatigue was assessed using the Krupp Fatigue Severity Scale (FSS), ${ }^{[9]}$ which consists of nine items each rated on a 7 point scale, which has 5 equidistant anchor points - strongly disagree (1), rare (2-3), sometimes (4), frequently (5-6), and strongly agree (7). The score is obtained by computing the average of the nine items with higher scores indicating increasing fatigue severity. Patients having a total FSS score of 36 and above are categorized as having fatigue and those who have a score $<36$ are categorized as not having fatigue. History of medication use such as antidepressants, sedatives, and antihypertensives, which might have contributed to fatigue, was recorded.

Depression was assessed using Beck's Depression Inventory $(\mathrm{BDI}),{ }^{[10]}$ which is a self rating questionnaire consisting of 21 groups of statements that evaluates the state of mood over the preceding 2 weeks. Higher scores $(\geq 17)$ indicate greater severity of depression.

Sleep quality was assessed using the Pittsburgh sleep quality index (PSQI), ${ }^{[1]]}$ which measures the subjective sleep quality during the preceding 1-month period. It consists of 19 self-rated questions and 5 questions rated by the bed partner. The 19 items are grouped into 7-component scores - sleep quality, latency, duration, sleep efficiency, disturbances, use of sleep medication, and daytime dysfunction. These component scores were added to a global PSQI score with a range from 0 to 21, higher scores indicating worse sleep quality. PSQI scores of above 5 were taken as abnormal.

Scores of depression, sleep disturbance, and fatigue were noted down using these scales in all patients and data was analyzed.

\section{Statistical analysis}

Statistical analysis was performed using SPSS version 15 software (SPSS Inc., Chicago, IL). Descriptive statistics included frequency, means, and standard deviation for quantitative variables such as age, age at first symptoms, number of episodes, EDSS and FSS scores, and duration of illness. All quantitative measures were treated with parametric statistical analysis such as Student's $t$-test, like; FSS, and BDI. Qualitative measures such as correlation of depression and sleep disturbance with associated fatigue were analyzed using Chi-square test.

\section{Results}

\section{Clinico-demographic profile}

This prospective study included 31 patients (6 male and 25 female). The age of patients in the study ranged from 16 to 50 years (30.1 \pm 9.1 years). The mean age at first symptom was $25.2 \pm 6.4$ years (range 14-39 years). The number of relapses (mean $\pm \mathrm{SD}$ ) was $4.7 \pm 3.6$ (range 1-14) in the study. The mean duration of illness was $4.9 \pm 4.4$ years (range $1-17$ years). The mean EDSS score in the study was $3.5 \pm 2.2$ (range 1-8).

The clinical presentation of MS patients was as follows: A total of 7 patients had clinical picture of 
quadriparesis (22.6\%), 15 patients had paraparesis (48.4\%), 18 patients $(58.1 \%)$ had sensory disturbances, 22 patients had visual problems (71\%), 18 patients had sphincter disturbances (58.1\%), 13 patients had diplopia (41.9\%), 10 patients had dysarthria (32.3\%), and 8 patients had ataxia (25.8\%).

\section{Sleep quality and depression}

Sixteen patients (51.6\%) reported poor sleep quality with PSQI scores of $>5$. The PSQI scores were significantly greater in the patients with fatigue as compared with those without fatigue indicating that poor sleep quality is associated with fatigue in MS $(P=0.005)$. Excessive daytime sleepiness was higher in the group of patients with fatigue as compared with those without fatigue but the difference was not statistically significant $(P=0.058)$. Sixteen patients $(16 / 31 ; 51.6 \%)$ had depression $(\mathrm{BDI} \geq 17)$. The BDI scores were also significantly higher in the fatigue group showing that severity of depression also strongly correlated with fatigue $(P=0.001)$.

\section{Profile of fatigue in patients with multiple sclerosis}

The mean fatigue score was $38.7 \pm 18.5$ (range 11-63). This score was above the cut-off value of 36 in the FSS. The prevalence of fatigue in the study was $58.1 \%(18 / 31)$. The mean age at first symptom was significantly greater in the group of MS patients with fatigue as compared with those without fatigue $(P=0.01)$ [Table 1].

\section{Discussion}

Many factors like depression, sleep quality, and disability affect fatigue associated with MS. ${ }^{[3]}$ This hospital-based prospective study was performed with 31 patients with MS. The objective was to observe prevalence of depression and sleep disturbances in MS patients and to observe if there is correlation between these factors and associated fatigue.

Poor sleep quality correlates strongly with severity of fatigue in patients with MS, which is believed to be due

$\begin{aligned} & \text { Table 1: Comparison between patients of MS with and } \\
& \text { without fatigue-sleep quality and depression }\end{aligned}$
\begin{tabular}{lccc}
\hline Factors & $\begin{array}{c}\text { FSS }<36 \\
(\boldsymbol{n}=13)\end{array}$ & $\begin{array}{c}\text { FSS-36 and } \\
\text { above }(\boldsymbol{n}=18)\end{array}$ & $\boldsymbol{P}$ value \\
\hline Age in years & $25.2 \pm 8.1$ & $33.5 \pm 8.2$ & 0.01 \\
Age at $1^{\text {st }}$ symptom (years) & $20.9 \pm 3.6$ & $28.3 \pm 6.2$ & 0.001 \\
EDSS & $2.7 \pm 2.4$ & $3.9 \pm 2.01$ & 0.131 \\
PSQI & $3.6 \pm 2.8$ & $8.1 \pm 4.7$ & 0.005 \\
Excessive daytime sleepiness (\%) & $1(7.7)$ & $7(38.9)$ & 0.058 \\
BDI & $6.9 \pm 7.1$ & $23.8 \pm 13.0$ & 0.001 \\
\hline
\end{tabular}

${ }^{*} \mathrm{FSS}$ : Fatigue severity scale, EDSS: Expanded disability severity scale, PSQI: Pittsburg's sleep quality scale, BDI: Beck's depression inventory to restless leg syndrome, sleep disordered breathing and circadian rhythm abnormalities. ${ }^{[12]}$ Up to $80 \%$ of MS patients suffer from fatigue with more than $60 \%$ poor sleepers, which leads to reduced quality of life. More than $50 \%$ of MS patients complain of chronic sleep disturbances resulting in daytime sleepiness, increasing fatigue, and depression. These patients have significantly longer sleep latency and increased use of sleep medications. ${ }^{[5]}$ Poor sleep and fatigue was seen less prevalent in our study but highlights the important comorbidities associated with MS.

Increase in sleep disturbance in Remitting Relapsing Multiple Sclerosis (RRMS) could be explained by the patho-physiology of illness. Demyelinated lesions and inflammatory responses from RRMS may disrupt the sleep-wake cycle by interfering with response of the suprachiasmatic nucleus of the brain. It has also been reported that women with RRMS have more daytime sleepiness, poorer sleep quality, and hypnotic medication use than do healthy women. ${ }^{[13]}$

Depression, night-time leg cramps, younger age, pain, female sex, fatigue, shorter duration of MS, and nocturia are all associated with disturbed sleep. ${ }^{[14]}$ Sleep disturbance in female patients is found to be more than in males with similar illness, other chronically ill population, or the general population..$^{[15]}$ In our study, patients with fatigue tended to be older as compared with patients without fatigue, had poorer sleep quality and had greater severity of depression according to the BDI scores. Comparison between genders for prevalence of depression and sleep disturbances is not possible in the present study as we had predominantly female patients $(80 \%)$.

As in the general population, major depressive disorder (MDD) is more prevalent in women with MS than in men. ${ }^{[16]}$ The precise etiology of the elevated rate of depression in persons with MS is not known, and a combination of psycho-social, neurobiological, and disease-related factors is likely. ${ }^{[17]}$ Depression in MS may be a psychological reaction to the uncertainty of living with an unpredictable chronic condition. ${ }^{[18]}$ The contribution of MS-related cognitive deficits and resulting social stress and problems with occupational performance could be other culprits. ${ }^{[19]}$ Recent stressful life events and chronic stress show an association with depression in persons with MS. ${ }^{[16]}$ Social support has a protective effect and women with MS reported less social support than men with MS, perhaps contributing to higher rates of depression in women. ${ }^{[20]}$ A direct or indirect association between MS-related neurobiological factors including altered immune function, central 
nervous system (CNS) lesions, and abnormalities in hypothalamic functioning is suggested as other reasons for the excess depression seen in MS patients. ${ }^{[17]}$ The results of studies that examine the association between neurobiological pathology and depressive symptoms are inconclusive. ${ }^{[21]}$ Both lesions in the right temporal region of the brain as well as lesions in specific regions of the left side of the brain have been associated with a greater incidence of MDD. ${ }^{[22,23]}$ The role of disease modifying therapies such as interferon also has come under scrutiny. Although there is evidence of an increased risk of depressive symptoms with interferon use, methodological differences in the studies, especially in how depression is defined and measured, preclude a definitive conclusion about this association. Other factors that have been examined for an association with depression are age and severity, course and duration of MS. ${ }^{[24]}$

Depressive symptoms fluctuate over time in MS patient but do not show a tendency to an overall significant increase or decrease as a whole. Younger age, longer time since diagnosis of MS, progressive forms of MS and greater extent of functional limitation are predictors of greater depressive symptoms in the initial phase. With the exception of functional limitation, which shows an association with depression at all time periods, these variables do not predict the changes in depressive symptoms over time. ${ }^{[25]}$

Prevalence of depression would vary with BDI cut-off scores. One study reported prevalence of depression as $19 \%$ in a population-based sample of patients with MS with the BDI cut-off score $>13$. It rose to $40 \%$ when BDI cut-off score was brought down to $10 .{ }^{[26]}$ Another study reported a prevalence of $42 \%$ for clinically depressive symptoms in MS. ${ }^{[27]}$ Most of the studies have reported significantly more prevalence of depression in MS patients as compared with the general population. In our study, depression was prevalent in more than half of the patients (cut-off point: 16, according to BDI). However, severity of depression was observed to be significantly greater in MS patients with associated fatigue.

\section{Conclusions}

Prevalence of depression and sleep disturbance is high in patients with MS as is the prevalence of fatigue. Our study showed significant correlation between depression and fatigue in MS patients. Similarly sleep quality was observed to be poor in more than half of the patients in the study, and was statistically significantly correlated with MS associated fatigue. Day time sleepiness was also higher in the group with associated fatigue but did not reach significant level.

\section{Limitations of the study}

The study had a relatively small sample size. A longitudinal study with larger sample size would provide more insight regarding correlation between depression, sleep disturbance, and associated fatigue.

\section{References}

1. Dahl OP, Stordal E, Lydersen S, Midgard R. Anxiety and depression in multiple sclerosis. A comparative population-based study in Nord-Trøndelag County, Norway. Mult Scler 2009;15:1495-501.

2. Kale N, Agaoglu J, Tanik O. Neuropsychiatric manifestations in multiple sclerosis: Correlation of fatigue and depression with disease progression. Neurol Res 2010;32:221-3.

3. Patrick E, Christodoulou C, Krupp LB; New York State MS Consortium. Longitudinal correlates of fatigue in multiple sclerosis. Mult scler 2009;15:258-61.

4. Johannsan S, Ytterberg C, Hillert J, Widen Holmquist L, von Koch L. A longitudinal study of variations in and predictors of fatigue in multiple sclerosis. J Neurol Neurosurg Psychiatry 2009;79:454-7.

5. Lobentanz IS, Asenbaum S, Vass K, Sauter C, Klösch G, Kollegger H, et al. Factors influencing quality of life in multiple sclerosis patients: Disability, depressive mood, fatigue and sleep quality. Acta Neurol Scand 2009;110:6-13.

6. Mills RJ, Young CA. The relationship between fatigue and other clinical features of multiple sclerosis. Mult Scler 2009;17:604-12.

7. Polman CH, Reingold SC, Edan G, Filippi M, Hartung HP, Kappos L, et al. Diagnostic criteria for multiple sclerosis: 2005 revisions to the McDonald Criteria. Ann Neurol 2005;58:840-6.

8. Kurtzke JF. Rating neurologic impairment in multiple sclerosis: An expanded disability status scale (EDSS). Neurology 1983;33:1444-52.

9. Krupp LB, Alvarez LA, LaRocca NG, Scheinberg LC. Fatigue in multiple sclerosis. Arch Neurol 1988;45:435-7.

10. Beck AT, Ward CH, Mendelson M, Mock J, Erbaugh J. An inventory for measuring depression. Arch Gen Psychiatry 1961;4:561-71.

11. Buysse DJ, Reynolds CF $3^{\text {rd }}$, Monk TH, Berman SR, Kupfer DJ. The Pittsburgh sleep quality index: A new instrument for psychiatric practice and research. Psychiatry Res 1989;28:193-213.

12. Moreira NC, Damasceno RS, Medeiros CA, Bruin PF, Teixeira CA, Horta WG, et al. Restless leg syndrome, sleep quality and fatigue in multiple sclerosis patients. Braz J Med Biol Res 2008;41:932-7.

13. Attarian HP, Brown KM, Duntley SP, Carter JD, Cross AH. The relationship of sleep disturbances and fatigue in multiple sclerosis. Arch Neurol 2004;61:525-8.

14. Bamer AM, Johnson KL, Amtmann DA, Kraft GH. Beyond fatigue: Assessing variables associated with sleep problems and use of sleep medications in multiple sclerosis. Clin Epidemiol 2010;2:99-106.

15. Bamer AM, Johnson KL, Amtmann D, Kraft GH. Prevalence of sleep problems in individuals with multiple sclerosis. Mult Scler 2008;14:1127-30.

16. Patten SB, Metz LM, Reimer MA. Biopsychosocial correlates of lifetime major depression in a multiple sclerosis population. Mult Scler 2000;6:115-20.

17. Goldman Consensus Group. The Goldman Consensus statement on depression in multiple sclerosis. Mult Scler 2005;11:328-37.

18. Dalton EJ, Heinrichs RW. Depression in multiple sclerosis: A quantitative review of the evidence. Neuropsychology 2005;19:152-8.

19. Gilchrist AC, Creed FH. Depression, cognitive impairment and social stress in multiple sclerosis. J Psychosom Res 1994;38:193-201.

20. Pakenham KI. Adjustment to multiple sclerosis: Application of a stress and coping model. Health Psychol 1999;18:383-92.

21. Siegert RJ, Abernethy DA. Depression in multiple sclerosis: A review. J Neurol Neurosurg Psychiatry 2005;76:469-75. 
22. Benesova Y, Niedermayerova I, Mechl M, Havlikova P. The relation between brain MRI lesions and depressive symptoms in multiple sclerosis. Bratisl Lek Listy 2003;104:174-6.

23. Feinstein A. The neuropsychiatry of multiple sclerosis. Can J Psychiatry 2004;49:157-63.

24. Feinstein A. Multiple sclerosis, disease modifying treatments and depression: A critical methodological review. Mult Scler 2000;6:343-8.

25. Beal CC, Stuifbergen AK, Brown A. Depression in multiple sclerosis: A longitudinal analysis. Arch Psychiatr Nurs 2007;21:181-91.

26. Gottberg K, Einarsson U, Ytterberg C, de Pedro Cuesta J, Fredrikson S, von Koch L, et al. Health-related quality of life in a population-based sample of people with multiple sclerosis in Stockholm County. Mult Scler
2006;12:605-12

27. Chwastiak L, Ehde DM, Gibbons LE, Sullivan M, Bowen JD, Kraft GH. Depressive symptoms and severity of illness in multiple sclerosis: Epidemiologic study of a large community sample. Am J Psychiatry 2002;159:1862-8

How to cite this article: Nagaraj K, Taly AB, Gupta A, Prasad C, Christopher R. Depression and sleep disturbances in patients with multiple sclerosis and correlation with associated fatigue. J Neurosci Rural Pract 2013;4:387-91.

Source of Support: Nil. Conflict of Interest: None declared.

Announcement

\section{iPhone App}

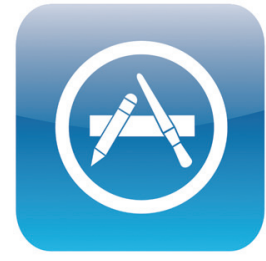

A free application to browse and search the journal's content is now available for iPhone/iPad. The application provides "Table of Contents" of the latest issues, which are stored on the device for future offline browsing. Internet connection is required to access the back issues and search facility. The application is Compatible with iPhone, iPod touch, and iPad and Requires iOS 3.1 or later. The application can be downloaded from http:// itunes.apple.com/us/app/medknow-journals/id458064375?ls $=18 \mathrm{mt}=8$. For suggestions and comments do write back to us. 